\title{
El paciente que padece un trastorno depresivo en el trabajo
}

\author{
The patient with depression at work
}

\author{
José Carlos Mingote Adán \\ Psiquiatra. Coordinador del Plan de Atención Integral al Profesional Sanitario Enfermo (PAIPSE) \\ Macarena Gálvez Herrer \\ Psicóloga. Plan de Atención Integral al Profesional Sanitario Enfermo (PAIPSE) \\ Pablo del Pino Cuadrado \\ Psiquiatra. Plan de Atención Integral al Profesional Sanitario Enfermo (PAIPSE) \\ $\mathbf{M}^{a}$ Dolores Gutiérrez García \\ Trabajadora Social. Plan de Atención Integral al Profesional Sanitario Enfermo (PAIPSE) \\ Correspondencia: \\ José Carlos Mingote Adán \\ PAIPSE. Pabellón 8. Facultad de Medicina \\ Ciudad Universitaria. 28040 Madrid. España
}

Resumen

La distinción entre sentimientos de tristeza normal, síndrome de desgaste profesional y los trastornos depresivos propiamente dichos, no siempre es fácil. En demasiadas ocasiones se carece de protocolos de actuación adecuados y las propias condiciones laborales impiden un abordaje satisfactorio e integral; es más, dichas condiciones pueden incluso actuar como desencadenantes, mantenedores o mediadores de muchos de estos problemas.

La depresión es una enfermedad como otra cualquiera y que nos puede pasar a cualquiera, pero en más de la mitad de los casos tiende a cronificarse y/o complicarse con otras enfermedades. Las consecuencias serán muy negativas, tanto en los trabajadores como en las organizaciones, si no se diagnostica de forma precoz y no se trata de forma efectiva. Con este objetivo, se revisa cada subtipo de trastorno, sus posibles efectos y reconocimiento en el trabajo, el abordaje de la persona afectada y el tratamiento.

Palabras clave: Salud mental, lugar de trabajo, depresión, trastorno adaptativo, Med Segur Trab 2009; 55 (214): 41-63

\begin{abstract}
|
The distinction among normal feelings of sadness, burnout and depressive disorders is not always easy to differentiate. In too many occasions, we lack the accurate procedures to deal with these problems and very often work conditions are an impediment to carry out a satisfactory and complete approach. These conditions can even originate, keep or mediate in many of the cases.

Depression is a disease as any other and anyone can have it, but more of the $50 \%$ of the cases tend to cronify or complicate with other diseases. If we don't do an early diagnosis to treat it in an effective way, the consequences will be very negative for workers and organizations. With this purpose we review each type of depressive disorder, its possible effects and recognition at work to approach an treat the ill person.
\end{abstract}

Key words: Mental health, workplace, depression, adaptative disorder, Med Segur Trab 2009; 55 (214): 41-63 


\section{INTRODUCCIÓN}

El manejo de los problemas de salud mental en el mundo laboral no es sencillo. En demasiadas ocasiones se carece de protocolos de actuación adecuados y las propias condiciones laborales impiden un abordaje satisfactorio e integral, es más, dichas condiciones pueden incluso actuar como desencadenantes o facilitadores de muchos de estos problemas. En un número anterior de esta revista tratamos el problema del paciente que padece un trastorno psicótico en el trabajo ${ }^{1}$, en esta ocasión, el objetivo del presente artículo es realizar una revisión sobre los diferentes trastornos depresivos, sus efectos en el ámbito laboral, la interacción con el mismo, y posibles líneas de tratamiento e intervención.

En el ámbito psicosocial, la depresión produce un gran sufrimiento en la persona afectada y en las personas de su entorno, con importantes repercusiones laborales y socioeconómicas, debido a la pérdida de productividad, al absentismo y a las posibles bajas laborales relacionadas. Una identificación y tratamiento adecuado, revierte estos efectos negativos de la depresión no tratada.

\section{¿En qué consisten los trastornos depresivos?}

La depresión es una palabra de uso común aunque puede tener diferentes significados. A menudo se utiliza para referirse a períodos pasajeros de tristeza o desánimo tras experimentar la pérdida de un ser querido, de la salud, de pertenencias personales, tras la frustración de expectativas deseadas, o ante dificultades cotidianas. En psiquiatría, las depresiones son trastornos mentales con síntomas y signos característicos, aunque no específicos, de suficiente intensidad y duración como para deteriorar el funcionamiento diario y la calidad de vida de la persona afectada.

Los habituales momentos o "días bajos" de cualquier persona se caracterizan por un sentimiento de tristeza "normal", leve, transitoria, asociada a pensamientos negativos en relación con problemas identificables tal y como indican Ramos y Cordero (2005) diferenciándola de la tristeza patológica (tabla 1).

Tabla I. Diferencias entre la tristeza normal y patológica según Ramos y Cordero (2005)

\begin{tabular}{|c|c|}
\hline Tristeza Normal: & Tristeza Patológica: \\
\hline — El motivo es siempre de carácter negativo. & $\begin{array}{l}\text { - El motivo no es siempre de carácter negativo, } \\
\text { puede tener carácter positivo, cualquier cambio. }\end{array}$ \\
\hline - El tiempo de aparición es casi inmediato. & - El tiempo de aparición es hasta 6 meses después. \\
\hline — La duración es adecuada al motivo. & — La duración es desproporcionada. \\
\hline — La intensidad adecuada al motivo. & — La intensidad es desproporcionada. \\
\hline $\begin{array}{l}\text { - El rendimiento no queda afectado significativa- } \\
\text { mente. }\end{array}$ & — El rendimiento queda afectado significativamente. \\
\hline $\begin{array}{l}\text { - La reactividad a estímulos externos permanece } \\
\text { prácticamente intacta. }\end{array}$ & $\begin{array}{l}\text { - La reactividad a estímulos externos queda dismi- } \\
\text { nuida o ausente. }\end{array}$ \\
\hline $\begin{array}{l}\text { - La vivencia de sentimientos se mantiene práctica- } \\
\text { mente intacta. }\end{array}$ & $\begin{array}{l}\text { - La vivencia de sentimientos queda disminuida o } \\
\text { ausente. }\end{array}$ \\
\hline $\begin{array}{l}\text { - El tipo de experiencia es conocida, psicológica- } \\
\text { mente "localizable". }\end{array}$ & $\begin{array}{l}\text { - El tipo de experiencia es cualitativamente diferente, } \\
\text { corporalizada, vaga, imprecisa. }\end{array}$ \\
\hline
\end{tabular}

Por otro lado, es importante diferenciar entre lo que sería un trastorno adaptativo de un trastorno del estado de ánimo propiamente dicho. El trastorno adaptativo es una reacción desadaptativa, caracterizada por una alteración del estado de ánimo que interfiere con la actividad personal y produce un deterioro significativo de la calidad de vida (ej. incumplimiento terapéutico); se asocia a un acontecimiento vital estresante reconocible, 
acontecido en los últimos tres meses previos y no cumple los criterios diagnósticos de otros trastornos específicos, aunque si se trata, puede prevenirse su ulterior desarrollo. Respecto al trastorno del estado de ánimo, éste incluye la depresión mayor, la distimia y el trastorno bipolar, que iremos desarrollando a lo largo del presente trabajo.

\section{Diagnóstico diferencial}

La sintomatología depresiva es la más frecuente junto con la ansiedad que observamos en los trastornos psíquicos y debe ser estudiada en el cortejo sintomático, el tiempo de aparición y la secuencia, considerando el contexto vital del paciente y su "historia de vida".

El primer reto es la diferenciación de las depresiones secundarias a enfermedad somática o formando parte de la sintomatología de ésta y las depresiones primarias.

Debemos descartar el consumo, abuso o abstinencia de tóxicos o fármacos. "El trastorno del estado de ánimo inducido por sustancias".

En los trastornos del estado de ánimo primario, hemos de precisar si forma parte de un episodio depresivo, si han existido otros episodios y si existe o ha existido previamente síntomas acoso-hipomaniacos. Son importantes las consecuencias en el abordaje farmacológico. Básicamente los episodios se diferenciarán en "unipolares" y "bipolares". (Trastorno Bipolar I, Trastorno Bipolar II y Trastorno Ciclotímico).

La duración y persistencia de los síntomas depresivos apuntan a trastornos distímicos.

El contexto vital reciente, la existencia de factores estresantes externos, los procesos de duelos forman parte de los trastornos depresivos reactivos o trastornos adaptativos. A veces su gravedad y duración llegan a conformar un estado depresivo mayor.

El manual de diagnóstico diferencial del DSM-IV nos propone un árbol de decisión (ver anexo).

\section{Depresión y trabajo}

Contar con un empleo tiene efectos protectores frente a la depresión, al proporcionar mayor seguridad económica, integración social y oportunidad para establecer relaciones y redes de apoyo social. El empleo constituye una respuesta de la sociedad a la necesidad de las personas de disponer de una base económica segura para crear una familia propia y una vida independiente.

A su vez, la depresión es la principal causa de discapacidad en el mundo ${ }^{3}$. Al menos un $20 \%$ de personas sufrirán una depresión a lo largo de su vida. En un año cualquiera, un 9,5\% de los adultos enfermarán por este motivo, lo que en España afecta a 450.000 personas. En un momento cualquiera de nuestra vida, uno de cada veinte adultos en edad laboral, estarán clínicamente deprimidos.

En Estados Unidos, la Corporación RAND ${ }^{4}$ señala que la depresión produce más bajas laborales que otras causas médicas tales como úlceras gástricas, hipertensión arterial, diabetes o artritis. En nuestro país, la VI Encuesta de Condiciones de Trabajo indica que el $22,5 \%$ de la población piensa que el trabajo está influyendo en su salud, y entre éstos, un 5,4\% señalan síntomas depresivos como la principal consecuencia ${ }^{5}$. Parece por tanto, que existe una doble direccionalidad e influencia recíproca que merece ser estudiada.

Figura 1. Interrelación trabajo y depresión

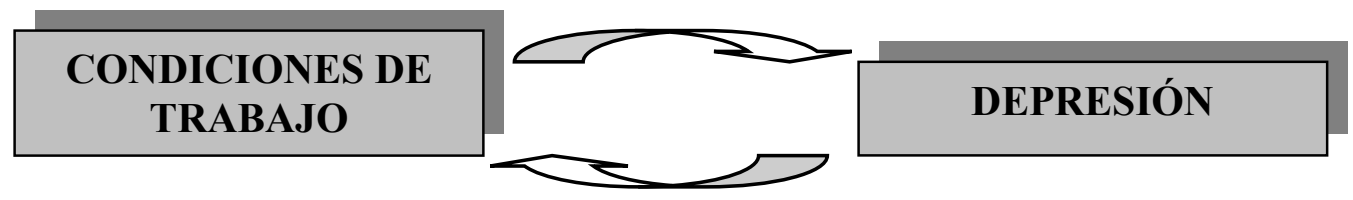




\section{EFECTOS DE LA DEPRESIÓN DE UNA PERSONA EN SU TRABAJO}

Se ha constatado la importancia del deterioro en los roles sociolaborales y en las relaciones interpersonales como consecuencia de la depresión, evaluando esta situación tanto de forma objetiva como subjetiva o percibida. En este último caso, destaca la insatisfacción y malestar experimentado en el trabajo y cómo, por el contrario, cuando la depresión mejora, se incrementa la satisfacción e incluso el rendimiento laboral de forma paralela a la disminución de los síntomas positivos.

Cuando existe una recurrencia depresiva, ésta se acompaña de un rápido empeoramiento del funcionamiento sociolaboral, referido tanto a la eficacia en la realización de las tareas como a la aparición de conductas de aislamiento y frecuentes conflictos interpersonales. En el trabajo, las personas con depresión:

- Están desmotivadas en mayor medida.

- Mantienen deseos de abandono profesional o retirada prematura

- Establecen peores relaciones personales con problemas de comunicación

- Se ven implicadas con mayor frecuencia en situaciones de conflicto laboral

- Disminuyen su rendimiento, capacidad de concentración y eficacia

Coyne $^{6}$ estudió cómo los pacientes con depresión inducen respuestas negativas de las personas con que se relacionan, desencadenando antipatía, sentimientos de incapacidad, tendencia a ser evaluados negativamente y a ser evitados. Las personas deprimidas suelen "ser deprimentes" en sus interacciones con los demás. Así por ejemplo, los cónyuges de personas con depresión tienen mayores tasas de trastornos mentales que los casados con personas sanas ${ }^{7}$. Este fenómeno puede deberse no sólo a los efectos adversos de la convivencia con una persona deprimida, sino también a otros efectos contribuyentes como un emparejamiento concordante y a otros factores estresantes secundarios.

La integración laboral es un componente clave para facilitar un autoconcepto positivo, autonomía, independencia e integración social del individuo; por ello, las personas con depresión suelen sobre-implicarse en el trabajo para neutralizar los sentimientos de incapacidad y culpa, e intentar mejorar su mala autoestima básica. Cuando la depresión se agrava se produce un cambio de actitud, incrementándose la irritabilidad y por tanto la conflictividad, disminuyendo la involucración con el trabajo e incrementándose los deseos de abandono y las conductas de absentismo.

\section{FACTORES PSICOSOCIALES DEL TRABAJO Y DEPRESIÓN}

Especialmente desde los años 70, son numerosos los estudios que asocian el estrés laboral con síntomas o trastornos depresivos ${ }^{8,12}$.

Concretamente, algunos trabajos de carácter longitudinal ${ }^{13}$ señalan factores psicosociales tales como la capacidad de control y autonomía sobre la tarea, la variedad y posibilidad de aprendizaje de nuevas habilidades y el apoyo social en el trabajo, como elementos que se relacionan de forma directa con un incremento de sintomatología depresiva a lo largo del tiempo. Esta pérdida de control sobre las propias actividades y los planes y metas profesionales a largo plazo, podrían ser explicados desde los modelos teóricos que explican la depresión en un marco de indefensión aprendida y progresiva pérdida de control.

El estrés laboral tiene una clara influencia sobre la sintomatología afectiva, especialmente si las demandas psicológicas del trabajo se asocian a un bajo nivel de control sobre las mismas y a escaso apoyo social ${ }^{14,15}$.

Estos datos correlacionan con menores tasas de suicidio en profesiones de carácter más autónomo, tales como arquitectos o ingenieros y con una mayor incidencia en profesiones de alto nivel de estrés como las asistenciales. El riesgo mayor de suicidio se ha observado en médicos, seguidos por personal en situación de desempleo y enfermeras ${ }^{16}$. 
En cualquier caso, para todos los grupos profesionales, los problemas de pérdida o riesgo de pérdida en el trabajo pueden dar lugar a depresión. Dicha pérdida se puede focalizar en el control sobre la propia actividad, en cambios continuados de puesto de trabajo, en las tareas a realizar, en una posible pérdida de empleo, en recortes de plantilla, prejubilaciones no deseadas, etc. En definitiva, la depresión puede tener una etiología relacionada con factores laborales que suponen una pérdida de los ideales, expectativas y autoimagen profesional.

\section{Depresión mayor}

\section{a) Criterios diagnósticos}

Se trata de un síndrome depresivo de intensidad significativa como para alterar la percepción de salud de la persona afectada e interferir en su funcionamiento habitual. La palabra mayor no implica necesariamente una gravedad máxima ya que los episodios depresivos más frecuentes son de una intensidad moderada y el paciente puede mantener parcialmente su actividad habitual a expensas de realizar un gran esfuerzo y de experimentar un gran sufrimiento personal. Los cuadros clínicos más graves se caracterizan por la presencia de síntomas endógenomorfos o melancólicos y con síntomas psicóticos.

Los instrumentos más sensibles para el diagnóstico de la depresión, con una sensibilidad entre el 96-99\%, son dos preguntas:

- ¿Se ha notado triste o deprimido durante el último año?

- ¿Ha perdido interés por las cosas?

Aunque con una especificidad muy baja (50\%), por lo que tras la sospecha de depresión en un enfermo se debe explorar la presencia del síndrome depresivo. Los criterios del trastorno depresivo mayor según el DSM-IV son los siguientes (más de cinco síntomas durante más de dos semanas):

Estado de ánimo depresivo y/o

Perdida de interés o placer, de manera que son necesarios uno de estos dos criterios diagnósticos.

- Anorexia y adelgazamiento (más del 5\% en 1 mes) o aumento de peso.

- Insomnio o hipersomnia.

- Enlentecimiento o agitación psicomotriz.

- Pérdida de energía o fatiga.

- Sentimientos de inutilidad, culpa o autoreproches.

- Deterioro de la capacidad de concentración, de pensamiento o de indecisión.

- Ideas de muerte o suicidio.

- La intensidad de los síntomas interfiere de forma significativa en la vida del paciente.

- No es explicable por un trastorno orgánico ni por un estado normal de duelo.

Más allá de la preceptiva exploración del estado mental del paciente, es imprescindible tener en cuenta también, su experiencia subjetiva respecto a la enfermedad y a posibles tratamientos previos. Hacerlo así mejora la satisfacción del paciente y la relación médicopaciente, la adherencia al tratamiento y la eficiencia institucional.

\section{b) Causas}

La depresión es un trastorno psicobiológico complejo, de etiología multifactorial que se explica por la interacción de varios factores de riesgo como los genéticos, neurobiológicos, socio-ambientales y otros propios del desarrollo de cada persona.

Así, aunque hasta el 70\% del riesgo de padecer una depresión es debido a factores genéticos, el hecho de sufrir adversidades o carencias en la infancia está implicado de 
forma significativa en su padecimiento. Con frecuencia, los pacientes que presentan depresión mayor comunican haber sufrido malos tratos en la infancia, (sean negligencia de cuidados, abuso físico, emocional o negligencia emocional), sobre todo en los casos de inicio precoz y curso recurrente. En las mujeres con depresión, se dan mayores tasas de abuso sexual, en torno al $22 \%$ de ellas, que en el caso de los varones ronda el 9\%. Para ambos géneros, el factor de riesgo más importante es la exposición a situaciones vitales estresantes recientes. Así, por ejemplo, la inseguridad laboral, la exclusión social y la pobreza median en un mayor riesgo de sufrir trastornos depresivos y de ansiedad.

Entre los acontecimientos vitales estresantes más estudiados y que predisponen a la depresión son: la pérdida de uno de los progenitores antes de los 11 años y la pérdida del cónyuge, sobre todo cuando las experiencias adversas dañan la autoestima personal. Por ejemplo, las mujeres médicos tienen una probabilidad del $60 \%$ mayor que sus compañeros varones de desarrollar desgaste profesional (Burnout), mayor riesgo de sufrir depresión y una mayor tendencia al suicidio respecto a la población femenina general, según la excelente revisión de Pilar Arrizabalaga y Carme Valls-LLobet17. Esto se explica no sólo por la mayor vulnerabilidad de la mujer a la depresión, sino también porque la mujer médico sufre una importante discriminación en cuanto a promoción jerárquica, retribuciones salariales, acceso a la docencia y a la investigación y por tener un mayor riesgo de acoso psicológico y sexual en el trabajo.

\section{c) Efectos y reconocimiento en el trabajo}

La depresión mayor tiene muchos más efectos sobre los empleados y las organizaciones de los que habitualmente se reconocen, debido al no reconocimiento de los síntomas característicos y sus complicaciones más frecuentes. En el trabajo pueden ser evidentes el aislamiento de los compañeros y la evitación de situaciones como las reuniones de trabajo, por la intensidad del malestar depresivo. Además, el empleado con depresión no le encuentra sentido al trabajo, factor que influye en el descenso de su productividad y colaboración.

La persona con depresión puede tener, en ocasiones, alterado el juicio de realidad, tomar decisiones erróneas y tardías, e incluso participar de accidentes y posibles daños para su salud y la de terceros, ausentarse de forma injustificada de su puesto y desmoralizar al resto de compañeros de trabajo. Cuando la depresión no se reconoce de forma adecuada suele agravarse, ya sea de forma lenta o abruptamente, llegando a complicándose con conductas de riesgo tales como adicciones, accidentes o suicidio consumado.

\section{d) Gestión y orientación}

Se recomienda considerar los siguientes aspectos:

1. Establecer relación y hacer una confrontación adecuada: La intervención inicial debe hacerse en un entorno de privacidad, con respeto a la persona afectada, sin paternalismo y sin trasmitir sensación de superioridad. Se ha de transmitir la preocupación por el cambio percibido en los hábitos de trabajo y en su estado de ánimo.

2. Disposición de escucha: Será importante mantener actitudes de escucha activa, atendiendo a las verbalizaciones de la persona con posible depresión y mostrar disposición para su comprensión.

3. Expresión de apoyo: Se recomienda mantener actitudes de apoyo emocional, normalización (con frases del tipo "la depresión es una enfermedad como otra cualquiera...", "no se trata de un defecto o de un fracaso personal", "es algo que nos puede pasar a todos en algún momento de nuestra vida y para lo que existe tratamiento eficaz")

4. Ayuda profesional: Ofrecer la posibilidad de acceso a un especialista en salud mental para realizar un tratamiento eficaz. 


\section{e) Tratamiento}

En la entrevista de evaluación será importante descartar enfermedades médicas y posibles adicciones relacionadas con los síntomas depresivos.

Tratamiento médico: Tradicionalmente el abordaje se ha realizado con antidepresivos, incluyéndose a partir de los años 50 iproniazida e imipramina, y a partir de los años 80 los inhibidores selectivos de recaptación de la serotonina (ISRS), que en la actualidad se consideran el tratamiento de primera elección de la depresión, salvo en las formas graves y psicóticas con riesgo de suicidio o cuando existan otras patologías asociadas que así lo requieran. Los casos en los que deben tenerse en cuenta otras indicaciones terapéuticas como la terapia electroconvulsiva en régimen de hospitalización para garantizar la seguridad del enfermo.

Una vez que se ha iniciado la administración de un antidepresivo, el tratamiento pasa por varias fases: fase temprana (previa a la respuesta), fase de respuesta o de tratamiento agudo (entre 2 y 4 meses) que debe continuarse una vez lograda la remisión clínica durante 8-12 meses (fase de continuación) y fase de mantenimiento a largo plazo (entre los 5 años y el tratamiento indefinido) en casos crónicos y/o recurrentes (con antecedentes de tres o más episodios depresivos). En los casos de depresión refractaria, está indicado derivar al enfermo al servicio de salud mental que le corresponda donde se emplearán diferentes estrategias de potenciación terapéutica como la administración de carbonato de litio y la asociación de un segundo antidepresivo.

En relación con el tratamiento antidepresivo, deben tenerse en cuenta los siguientes conceptos principales:

1. Eficacia terapéutica: con dosis adecuadas y un tiempo correcto, el $80 \%$ de los pacientes se benefician de cualquier tratamiento de los anteriormente expuestos.

2. Rapidez de acción: la respuesta terapéutica se demora en general entre 2 y 6 semanas para la mayoría de pacientes.

3. Eficacia con el tratamiento prolongado: lograda la respuesta terapéutica debe prevenirse una recaída en los primeros seis meses y evitar otras recurrencias depresivas.

Tratamiento psicoterápico: La psicoterapia de apoyo debe utilizarse en todos los casos para ayudar al paciente a restablecer su mejor nivel posible de funcionamiento psicológico, mejorar sus capacidades de afrontamiento, la calidad de vida individual y prevenir ulteriores recaídas.

Las diferentes perspectivas han demostrado su eficacia en el tratamiento de la depresión. Desde un enfoque psicodinámico, se afrontarán los conflictos del paciente implicados en el proceso depresivo. Desde una perspectiva interpersonal, cobrarán especial importancia los patrones de relación del paciente con las personas de su entorno (incluido el ámbito laboral). Desde un enfoque cognitivo-conductual, se trabajarán la triada cognitiva de pensamientos negativos sobre sí mismo, el mundo y el futuro, los esquemas mentales disfuncionales y los pensamientos irracionales, además de buscar refuerzos positivos en la conducta del paciente mediante la programación de actividades gratificantes, el entrenamiento en habilidades sociales o en solución de problemas, en función de las necesidades individuales de la persona.

\section{f) Reincorporación laboral}

La reincorporación al trabajo dependerá de la severidad del daño y de la pérdida de funcionalidad más que del diagnóstico en si mismo.

Se estima que al menos el $80 \%$ de las personas que sufren alguna de las formas más graves de depresión, mejoran de forma significativa con el tratamiento, lo que significa que la mayoría de ellas volverán a alcanzar su nivel normal de funcionamiento previo, en un período de dos a tres meses ${ }^{18}$, si bien en ocasiones este proceso se puede demorar en el tiempo. 


\section{Distimia}

\section{a) Criterios diagnósticos}

El término distimia, que etimológicamente significa mal humor, se utiliza para describir a las depresiones de intensidad moderada, curso crónico y/o fluctuante, que con frecuencia se inician antes de los 20 años en personas con antecedentes depresivos.

Se trata de un diagnóstico de baja precisión y que con frecuencia coexiste con otros diagnósticos psiquiátricos en el mismo paciente como síndromes ansiosos y trastornos de la personalidad. Se caracteriza por la existencia de un estado de ánimo deprimido la mayor parte del día, la mayoría de los días, durante mínimo 2 años.

- Presencia de dos o más de estos síntomas:

1. Perdida o aumento de peso.

2. Insomnio o hipersomnia.

3. Falta de energía o fatiga.

4. Baja autoestima.

5. Dificultades para concentrarse o tomar decisiones.

6. Sentimientos de desesperanza.

- No ha habido ningún episodio depresivo mayor durante los primeros 2 años (1 año para niños y adolescentes.). En el caso de que exista un trastorno depresivo mayor superpuesto, se realizaran ambos diagnósticos.

- Nunca ha habido un episodio maniaco, episodio mixto o hipomaniaco y no se han cumplido los criterios de trastorno ciclotímico.

- La alteración no aparece exclusivamente en el transcurso de un trastorno psicótico crónico, como son la esquizofrenia o el trastorno delirante.

- Los síntomas no son debidos a los efectos de una sustancia o enfermedad médica.

- Los síntomas generan gran malestar clínicamente significativo en la vida del paciente.

- Importante especificar si es de inicio temprano (antes de los 21 años) o de inicio tardío (de los 21 años en adelante) y si existen síntomas atípicos.

- Este subtipo, no tiene las características endógenas ya descritas y podemos encontrar los siguientes elementos diferenciales:

- Se trata de un trastorno crónico, con un comienzo insidioso (adolescencia) y con un curso fluctuante o intermitente.

- Los síntomas que refieren los pacientes son poco específicos y muy variables, menos intensos que en la depresión mayor.

- Antecedentes familiares de trastornos de ansiedad y depresión.

- Peor respuesta al tratamiento antidepresivo y alta tasa de respuesta al placebo.

- Forma de depresión que suele asociarse con determinadas situaciones ambientales estresantes, (pérdidas significativas, desgaste profesional, etc.), así como con la presencia de ciertas características de personalidad (obsesividad, neuroticismo elevado, etc.), que interactúan con factores neurobiológicos determinados genéticamente.

- Mayor comorbilidad que en las depresiones melancólicas con trastornos de la personalidad, trastornos de ansiedad y abuso de sustancias, que agravan su pronóstico.

\section{b) Causas}

Se ha constatado en varios estudios epidemiológicos, neuroendocrinológicos y electroencefalográficos, la existencia de una continuidad entre las depresiones leves y 
moderadas y las formas endogenomorfas más graves, motivos por los que los sistemas actuales de clasificación de los trastornos mentales han abandonado la dicotomía neurótico-endógeno.

La distimia se asocia con varios tipos de trastornos neuróticos de personalidad, que junto con situaciones ambientales estresantes dan lugar a cuadros depresivos que tienden a cronificarse por la ineficacia de los mecanismos de afrontamiento del estrés y el impacto de las situaciones conflictivas que padecen estos pacientes.

\section{c) Efectos y reconocimiento en el trabajo}

El reconocimiento de la distimia puede ser especialmente difícil al tratarse de varias alteraciones de baja intensidad sintomática y larga duración temporal, por lo que con frecuencia se confunde con la tristeza "normal" o el carácter "habitual" de la persona.

La distimia puede manifestarse con quejas físicas y bajas médicas excesivas, sin hallazgos objetivos de enfermedad médica alguna que las explique. La persona distímica puede parecer negativa, irritable, desinteresada e hipersensible a las críticas, con tendencia al aislamiento y a ser poco colaboradora. Puede expresar pérdida de la capacidad de disfrutar y frecuentes sentimientos de vacío crónico.

En ocasiones, el trabajador con distimia puede sentirse sobrecargado de trabajo e incluso discriminado por el superior jerárquico, así como verse implicado en repetidos conflictos con sus compañeros de trabajo.

\section{d) Gestión y orientación}

Al tratarse de una depresión de baja intensidad y larga duración puede confundirse con más facilidad con un problema de personalidad o con una debilidad o falta de carácter, con frecuente auto-inculpación de forma irrealista.

Se seguirán las mismas recomendaciones indicadas en el caso de la depresión mayor.

\section{e) Tratamiento}

Se recomiendan sesiones de psicoterapia semanal y tratamiento antidepresivo con ISRS como tratamiento de primera elección.

Aunque la mayor parte de los fármacos antidepresivos han demostrado ser efectivos en el tratamiento de la distimia, se prefieren los ISRS en las mismas dosis recomendadas para la depresión mayor, y al menos durante dos o más años. Según los estudios disponibles, el tratamiento farmacológico reduce la clínica depresiva, aunque la combinación de antidepresivo con psicoterapia cognitiva e interpersonal ha resultado la mejor opción, al aumentar los efectos del antidepresivo, con mejoría del control emocional y de la autoeficacia. De esta forma se ayuda a potenciar el cumplimiento terapéutico, a tratar la frecuente comorbilidad asociada y los problemas psicosociales, mitigando sus consecuencias y facilitando la resolución de los problemas; así como prevenir la aparición de nuevos episodios.

\section{f) Reincorporación laboral}

El abordaje psicoterápico será importante para una adecuada reincorporación laboral por lo que se aconseja mantener la flexibilidad necesaria para su seguimiento.

Será necesario el entrenamiento en el manejo y control de los posibles estresores laborales y el ajuste de expectativas, a fin de que la excesiva sensibilidad y criticismo presente en estos pacientes no sea foco de posibles conflictos laborales o que la presencia de los mismos acentúe la sintomatología distímica. 


\section{Trastorno bipolar}

\section{a) Criterios diagnósticos}

Los trastornos bipolares se caracterizan por la coexistencia de episodios maniacos, hipomaniacos o estados mixtos, a la vez que por la presencia o historia de episodios depresivos mayores. Se clasifican en:

- Trastorno bipolar I: caracterizado por sufrir un o más episodios maniacos o mixtos junto con episodios depresivos mayores.

- Trastorno bipolar II: caracterizado por la existencia de uno o más episodios depresivos mayores junto con al menos un episodio maniaco.

- Trastorno ciclotímico: se caracteriza por más de dos años de numerosos periodos de síntomas hipomaniacos y de síntomas depresivos que no cumplen los criterios para un episodio depresivo mayor.

Habitualmente se tardan unos diez años para que un paciente con trastorno bipolar sea correctamente diagnosticado, debido a la dificultad para identificar los síntomas de la enfermedad. Este retraso en recibir el diagnóstico y el tratamiento adecuado hace que el paciente tenga un peor pronóstico, con deterioro de su calidad de vida y padecer nuevas complicaciones. Es infrecuente su aparición por encima de los 50 años y la manía que aparece por primera vez después de los 65 años suele tener una etiología orgánica, habitualmente asociada con afectación vascular cerebral.

El perfil típico del paciente bipolar suele ser una persona joven de entre 20 y 30 años que en poco tiempo experimenta alteraciones de la conducta normal, que se torna excéntrica o grandiosa, con necesidad disminuida de dormir, estado de ánimo excesivamente elevado, etc.

\section{b) Causas}

El trastorno bipolar es bastante común, estimándose que su prevalencia vida es del 1,6\% en la población general (entre un 1 y un 3\%), lo que implicaría que en nuestro país unos 600.000 españoles van a sufrir la enfermedad en algún momento de su vida. Ocupa el sexto lugar entre las enfermedades más incapacitantes y es el trastorno mental que con más frecuencia se asocia con el consumo de sustancias adictivas (entre el 35-50\% de los pacientes). El riesgo de suicidio es muy alto: entre un $25-50 \%$ de los pacientes llevan a cabo tentativas de suicidio y el $19 \%$ lo consuman.

Según estudios familiares, el riesgo de padecer la enfermedad entre los familiares de primer grado de probandos con trastorno bipolar tipo I, es 7 veces mayor respecto a la población general, así como cifras superiores de depresión mayor y de trastorno bipolar II, los cuales tienen también un mayor riesgo de sufrir este mismo tipo de trastorno, lo que indicaría su independencia del grupo de trastorno bipolar I. Por otro lado, al igual que ocurre con en el trastorno depresivo mayor, se ha demostrado que diferentes acontecimientos vitales estresantes pueden desencadenar los primeros episodios del trastorno bipolar en mayor medida que en las descompensaciones posteriores: el estrés asociado a la primera descompensación del trastorno del estado de ánimo podría provocar la sensibilización de los sistemas neurobiológicos reguladores hasta crear un estado de ánimo inestable de forma cíclica.

\section{c) Efectos y reconocimiento en el trabajo}

Un episodio de manía suele ser una experiencia muy impactante y perturbadora, a menudo presenciada por los compañeros de trabajo, existiendo así la posibilidad de que se genere con ello un clima de inquietud y problemas interpersonales ante la situación; ya sea ésta un episodio de grandiosidad extrema, de violencia y/o de rechazo de las normas de conducta. Existe además peligro de daño a si mismo y/o a los demás por la escasa percepción de riesgo. 
Otras veces, los cambios de humor en breves periodos de tiempo (días u horas), pueden dejar sorprendidos y asustados a los compañeros del paciente. En ocasiones, estos "empleados enfermos" pueden resultar beneficiosos a las empresas durante cierto tiempo con su entusiasmo y optimismo característico. Será más adelante, cuando se hagan patentes las equivocaciones, los errores de juicio, la irritabilidad y la agresividad descontrolada.

En el caso del Trastorno Bipolar tipo II, las alteraciones de conducta son más leves y suelen confundirse con una personalidad simpática y carismática, creativa y muy productiva; aunque también pueden dispersarse, ser impulsivos y poco eficientes.

\section{d) Gestión y orientación}

La persona con manía o hipomanía tiene escasa o nula conciencia de enfermedad mental y suele carecer de motivación para pedir ayuda profesional.

Cuando el comportamiento laboral del empleado con este trastorno llega a hacerse evidentemente errático y poco efectivo, con riesgo para su propia seguridad o la de terceros, la empresa debe requerir una intervención voluntaria o involuntaria de carácter psiquiátrico.

En general, cuando éstos pacientes recuperan la estabilidad del humor pueden reincorporarse al trabajo y rendir igual que antes, debiendo asegurar que toma la medicación y acude a psicoterapia de forma continuada.

\section{e) Tratamiento}

Serán necesarios estabilizadores del ánimo de forma indefinida, siendo el carbonato de litio el tratamiento de primera elección. Pueden utilizarse también ácido valproico, carbamazepina y lamotrigina.

Los objetivos psicoterapéuticos se dirigirán hacia el incremento del autoconocimiento, el aprendizaje de estrategias adaptativas eficaces y la psicoeducación sobre el trastorno y prevención de sus consecuencias, tanto en el propio paciente como en su entorno más cercano, siendo en ocasiones recomendable la terapia familiar y de pareja.

\section{f) Reincorporación laboral}

La baja laboral dependerá más de la gravedad del trastorno que del tratamiento en sí mismo, y puede requerir desde cuatro o seis semanas hasta cuatro o seis meses.

En la reincorporación será importante considerar la posibilidad de flexibilizar el horario de forma que se facilite el tratamiento y cumplimiento de los requerimientos psicoterapéuticos.

\section{Depresión de inicio tardío}

El creciente envejecimiento de la población trabajadora nos impulsa a tratar un caso de depresión con frecuencia olvidado, la depresión de inicio tardío. Se trata de un trastorno en ocasiones confundido con otros problemas relacionados con aspectos evolutivos de las funciones neurovegetativas y mentales de la edad adulta, donde pueden darse coexistencia de síntomas y para el que el diagnóstico diferencial no es sencillo.

Un ejemplo cercano lo tenemos entre la profesión médica, donde existe un elevado porcentaje de facultativos de más de 50 años. Concretamente, en Madrid, el 56\% de los profesionales rebasan esa edad, el 50\% en Aragón, el 46\% en Castilla y León y el 45\% en Asturias. Sin embargo, son escasos los estudios que relacionan aspectos laborales entre estos profesionales con depresión de inicio tardío.

\section{a) Criterios diagnósticos}

Se trata de una forma de trastorno depresivo caracterizada por su inicio tardío, por su asociación con varios factores de riesgo cardiovascular, con deterioro cognitivo leve 
(apreciable a través de pruebas neuropsicológicas), así como con hallazgos característicos en las pruebas de neuroimagen, que predice demencia vascular en los años posteriores, aunque también permite la prevención de esta última patología.

El estudio de la depresión vascular plantea numerosos problemas metodológicos, y establecer la etiología vascular de la misma es imposible con datos clínicos, sin hacer estudios prospectivos. Existe una elevada frecuencia de patología mixta, dado que en los pacientes de edad avanzada coexisten habitualmente múltiples enfermedades (diferentes tipos de lesiones cerebrovasculares, neurodegenerativos, enfermedad coronaria, etc.) que interactúan entre si para producir el síndrome depresivo. La mala salud física es un factor de riesgo para la depresión de inicio tardío.

Por otra parte, su validez diagnostica es cuestionada, sobre todo la existencia de un patrón clínico definido que la diferencie de otros trastornos.

\section{b) Causas}

La relación entre depresión y enfermedades cardiovasculares (ECV) puede explicarse de cuatro formas posibles no excluyentes entre si: (1) la depresión como reacción psicológicamente comprensible ante la ECV; (2) la depresión como proceso explicable de forma secundaria al daño isquémico cerebral; (3) la depresión como factor de riesgo para la ECV y (4) como una asociación al azar.

La etiología de la depresión vascular es multifactorial:

1. Factores biológicos:

- Severidad del daño vascular cerebral, que es el que produce la desorganización de los circuitos neurales y de los sistemas neuroquímicos que participan en la regulación del humor.

- La hipertensión arterial no complicada produce cambios en la actividad eléctrica cerebral en paralelo con la disminución del flujo sanguíneo cerebral y del incremento de la excitabilidad neuronal, daño vascular e isquemia neuronal.

- Especificidad regional.

- Vulnerabilidad genética: antecedentes familiares de arteriosclerosis.

- La depresión se asocia a una elevada actividad autonómica simpática con incremento de la actividad plaquetaria, así como una excesiva secreción de glucocorticoides.

- Mayor expresión de marcadores de inflamación vascular.

2. Factores psicosociales:

- Exposición a mayor número de acontecimientos vitales estresantes e inadecuados mecanismos para el afrontamiento del estrés.

- Insuficiencia de soporte psicosocial y de recursos económicos, y aislamiento social.

- Elevado neuroticismo.

\section{c) Efectos y reconocimiento en el trabajo}

En estos casos son también aplicables las mismas consideraciones que en trabajadores más jóvenes, e igualmente requieren su detección temprana y un tratamiento eficaz para la prevención de posibles complicaciones como el suicidio (en especial en varones de edad avanzada y sin apoyo familiar).

\section{d) Gestión y orientación}

En los casos de depresión de inicio tardío, es especialmente importante la evaluación médica y el despistaje precoz de varias enfermedades asociadas, como son frecuentemente la hipertensión arterial, diabetes y síndrome de apnea del sueño. Si estas alteraciones no se tratan con efectividad, van a imposibilitar una recuperación funcional completa. 
La edad avanzada y el sexo femenino son conocidos factores predictivos de evolución crónica y/o de recurrencia, sobre todo cuando se asocian a situaciones estresantes acumuladas.

\section{e) Tratamiento}

1. Del síndrome depresivo con antidepresivos (nortriptilina y trazodona) así como TEC. La respuesta al tratamiento es peor que en otros tipos de depresiones, con mayor refractariedad al tratamiento medico, en relación con el deterioro de las funciones ejecutivas.

2. Reducción de la lesión, con bloqueadores de los canales del calcio, antagonistas del receptor de NMDA, inhibidores de la síntesis de glutamato, etc.

3. Control de los factores de riesgo vascular (HTA, dislipemias, diabetes).

Desde una perspectiva psicoterapéutica, el trabajo se orientará a la psicoeducación del paciente en su trastorno, la programación de actividades gratificantes, al autorefuerzo $\mathrm{y}$ al apoyo psicológico para el manejo de dificultades.

\section{f) Reincorporación laboral}

La reincorporación laboral es más tardía menos frecuente que en pacientes de menor edad, y con frecuencia está condicionada por limitaciones cognitivas, deterioro intelectual y por otras patologías médicas asociadas con cierta frecuencia.

\section{Trastorno adaptativo}

\section{a) Criterios diagnósticos}

Se trata con frecuencia de un diagnóstico provisional difícil de delimitar porque no incluye un perfil sintomático específico donde es difícil discernir entre elementos "adaptativos" y "desadaptativos".

Se habla de trastornos del estado de ánimo en referencia a desarrollos maladaptativos que incluyen sintomatología emocional y comportamental como respuesta a un estresor psicosocial identificable (durante los tres meses siguientes al mismo). El síndrome clínico supone un malestar mayor de lo esperable con deterioro significativo de la actividad sociolaboral y de la salud personal (conductas de riesgo, patologías psicosomáticas, etc.) sean de forma aguda (menos de seis meses) o crónica (más de seis).

Se da entre un $5 \%$ y un $20 \%$ de los enfermos psiquiátricos ambulatorios. Los dos factores discriminativos son baja autoestima y estado de ánimo depresivo con respecto a los no casos.

- Trastorno del estado de ánimo debido a una enfermedad médica: se caracteriza por una persistente alteración del estado de ánimo que puede explicarse como efecto fisiopatológico directo de una enfermedad médica reconocible.

- Trastorno del estado de ánimo inducido por sustancias: la alteración del estado de ánimo se explica como consecuencia del efecto fisiopatológico de una droga, medicamento o de otro tratamiento médico anterior.

- Trastorno del estado de ánimo no especificado: se utiliza para codificar los trastornos con síntomas afectivos que no cumplen los criterios para ninguno de los grupos anteriores.

\section{b) Causas}

En adultos, los estresores más comunes que están en el origen de los trastornos adaptativos son los problemas conyugales (25\%), procesos de separación o divorcio (23\%), mudanza (17\%), problemas de carácter económico (14\%) y dificultades de carácter laboral (9\%). 
Entre estas últimas cabe señalar algunas tales como la vivencia de episodios de violencia en el trabajo, situaciones de acoso o abuso emocional, cambios de trabajo o pérdida del mismo.

Otra situación estrechamente ligada a los trastornos adaptativos es el desgaste profesional o burnout. Entre las diversas conceptualizaciones de este síndrome, una de las más utilizadas ha sido la procedente de la factorialización de un cuestionario, el "Maslach Burnout Inventory" (MBI) de Maslach y Jackson"1, caracterizándolo por la percepción de pérdida de recursos emocionales para enfrentarse al trabajo (agotamiento emocional), el desarrollo de actitudes negativas, de insensibilidad y cinismo hacia los receptores del servicio prestado (despersonalización) y por una tendencia a evaluar el propio trabajo de forma negativa, con sentimientos y apreciaciones de baja autoestima profesional (falta de realización personal).

\section{c) Efectos y reconocimiento en el trabajo}

El malestar o el deterioro de la actividad asociado a los trastornos adaptativos se manifiesta frecuentemente a través de una disminución del rendimiento en el trabajo, de la motivación y de una reducción de las relaciones sociales.

En el caso concreto del desgaste profesional, los estudios de los últimos años (de forma prioritaria en Europa), plantean tendencias explicativas que están impulsando el desarrollo de una nueva generación de instrumentos de evaluación cuyo objetivo es superar algunas limitaciones conceptuales y psicométricas del MBI tales como la evaluación del agotamiento cognitivo y físico, no sólo emocional; la consideración de la despersonalización como un factor de distanciamiento no sólo hacia el paciente sino hacia la profesión en general; el estudio de la falta de realización personal o eficacia como un posible consecuente del desgaste profesional (pero que no forma parte del síndrome); y la tendencia a evaluar aspectos de carácter cognitivo-motivacional como la pérdida de expectativas o la ilusión en el trabajo, elementos mencionados desde los primeros modelos teóricos de desgaste profesional, pero que sólo se han considerado para su evaluación en algunos de los cuestionarios más recientes ${ }^{20}$.

\section{d) Gestión y orientación}

Igual que en los otros casos, aunque debe considerarse la naturaleza transitoria y el buen pronóstico general de estos trastornos.

En ocasiones, en función del estresor, puede ser necesario una reestructuración del puesto de trabajo o una reubicación de la persona que le separe temporalmente del foco estresor.

\section{e) Tratamiento}

Se recomienda consejo terapéutico e intervenciones psicoterapéuticas para el aprendizaje de la reducción del estrés, afrontamiento del mismo, ajuste de expectativas profesionales y técnicas para mejora de la autoestima.

Cuando el estresor es de carácter interpersonal, el entrenamiento en habilidades de comunicación, asertividad y competencias emocionales, incrementará los recursos personales del trabajador.

\section{f) Reincorporación laboral}

La evolución en adultos es buena, siendo en personas jóvenes (especialmente adolescentes) donde en ocasiones es predictor de otros trastornos psiquiátricos.

\section{CASO CLÍNICO}

Mujer médico de 45 años de edad, soltera, con ejercicio profesional como médico de familia desde 1986. Enviada desde el Servicio de Prevención de Riesgos Laborales de su 
Área Asistencial para valoración diagnóstica y tratamiento por padecer un trastorno depresivo-ansioso de 6 meses de evolución.

\section{Estado mental actual}

La paciente se queja de dolores lumbares y cefaleas, nerviosismo, insomnio de conciliación, pérdida de apetito, desánimo y llanto fácil, sobre todo al recordar varios incidentes recientes que ha tenido con algunos pacientes que la han insultado al negarse a hacerle las múltiples recetas que le había solicitado, con actitud agresiva y despectiva: "tuve que aguantar el chaparrón de improperios y de insultos como "tu me tienes que hacer estas recetas porque el seguro es mío", "usted es una inútil y usted me tiene que recetar lo que le pido porque lo necesito". "Salió de la consulta dando un portazo, y yo me relajé y me puse a llorar con impotencia, me sentí despreciada de forma inmerecida, además no puedo quitármelo del cupo, aunque él puede cambiarse de médico cuando quiere (y el cliente siempre tiene razón). En realidad, yo soy la que le atiende ahora después de hacer 3 cambios de médico". Carmen llora sin poder contenerse al recordarlo y sentir la desprotección e incomprensión del sistema sanitario. Asocia después con otro paciente que se enfadó también con ella por no autorizar de forma inmediata su solicitud de derivación a otro especialista y refiere que el paciente le gritó: "para eso pago, tú me tienes que mandar al especialista porque yo te lo digo. Eres una nulidad, para eso pago, sino sabes ser médico dedícate a otra cosa...".

Tras ser evaluada, reconoce que lleva tiempo pasándolo muy mal en el trabajo: "he tenido que ir dejando de hacer cosas para sobrevivir (llora de nuevo), trato de verles con detenimiento, y si no puedo me frustro, y tengo que tomar Tranxilium esporádicamente sin control por parte de ningún especialista (reconoce la automedicación de forma prolongada). Ahora lo llevo peor desde hace 6 meses, que se me murió un paciente, le valoré bien pero la familia estuvo muy agresiva conmigo. No sé que puedo hacer, aparte de intentar olvidarme del trabajo, buscar ayuda psicológica y aceptar sentirme como paciente y no como médico, o mejor dicho, como médico-paciente”. Además, Carmen reconoce que en los últimos meses tiene un persistente estado de ánimo depresivo, se siente enferma de cansancio, dolores de cabeza, falta de apetito y pérdida de 6 kilos de peso, desmotivación, tendencia al aislamiento y frecuentes fallos de memoria para hechos recientes, despistes que le han notado sus compañeros de trabajo, aunque no habían querido darle mayor importancia. Así mismo, refiere alteraciones del sueño con rumiaciones negativas, desesperanza y deseos pasivos de muerte, aunque no ideas de suicidio.

\section{Antecedentes Personales}

Destaca haber sufrido episodios de angustia en los primeros años de la carrera junto con úlcera duodenal. La paciente siempre ha sido una persona sana, salvo en una ocasión, hace 10 años que estuvo un mes de baja debido a una infección gastro-intestinal.

\section{Antecedentes familiares}

No presenta antecedentes psicopatológicos familiares.

\section{Biografía}

Es la mayor de 6 hermanos. Mantiene buenas relaciones con familia de origen, aunque se queja de no tener suficiente tiempo para poder realizar sus aficiones después de trabajar.

Los padres se casan estando la madre embarazada de Carmen. Ese año el padre emigra para trabajar, mientras la paciente y su madre, embarazada de nuevo, se quedan viviendo en casa de la familia paterna, esperando que nazca su segundo hijo. La familia después del nacimiento se reúne con el padre. El padre pertenecía a un estatus socioeconómico superior y la madre era despreciada por la familia de su marido al ser considerada de inferior posición por ser hija de un campesino. 
Por motivos de trabajo llegan a cambiar hasta 5 veces de ciudad. Los frecuentes cambios de domicilio hacen que Carmen no pueda mantener continuidad con sus amigos de infancia. A pesar de esto, tiene buenos recuerdos de esta etapa de su vida. La familia vuelve a Madrid cuando Carmen tiene 12 años. Al reanudar sus estudios en esta ciudad, se sintió discriminada por sus compañeros. Su nivel de conocimientos era superior y no tenía las mismas inquietudes que las chicas de su edad. Su gran preocupación era el estudio. Siempre ha sido muy reservada, responsable y autoexigente consigo misma.

Descripción del padre: "Siempre tenía razón, muy autoritario gritaba mucho a mi madre y a nosotros. A veces pegaba, sobretodo, a mi hermano. Siempre que gritaba yo tenía miedo que me pegase. Aquella situación me daba miedo y rabia. "Era muy exigente con mis estudios, en una ocasión me echó de casa por no saberme la tabla de multiplicar."

Descripción de la madre: "Siempre ha sido una mujer muy callada, nunca respondía a mi padre." "Siempre sacrificada, disponible para los demás, nunca se ha valorado." "Nos ha educado para pedir perdón por nada y dar las gracias por todo." "Siempre nos decía: "Tenéis que ser independientes para que no tengáis que deber nada a nadie."

Su vocación de médico nace siendo muy pequeña, siempre deseó tener una profesión de ayuda a los demás. Considera que ha recibido más facilidades que sus hermanos a la hora de estudiar, mientras que a su hermano le ha faltado apoyo en este aspecto: "Mi padre le descalificaba por sacar malas notas y le comparaba conmigo". "Siempre me ha preocupado mucho tratar a las personas con equidad, por eso me molesta tener que dar más a los pacientes exigentes y menos a los que no lo son".

Se independizó cuando terminó sus estudios. Pasó varios años trabajando en otra ciudad diferente y después volvió a Madrid. Conserva una buena amiga de la carrera, pero vive fuera y se ven poco. Una vez al año algunos compañeros de trabajo y amigos que conoció cuando trabajó fuera, organizan un fin de semana en el que se ponen al día de sus vidas.

Su gran amor lo vivió con 24 años. "Inicia el relato diciendo: "Era mi alma gemela". Era un artista que conoció en el trabajo. La familia se opuso a esta relación. Tenía que verle a escondidas y la oposición de su familia le producía gran sufrimiento. Carmen sabía que era un bohemio y que no duraría toda la vida, pero decidió vivir la relación. Duró un año. En una visita que le hizo, aprovechando que tenía familia en la misma ciudad donde su novio pasaba varios meses al año, descubrió que le era infiel. Su padre la descalificó, la humilló, e insultó por esta relación: "Ninguno de los chicos con los que he salido, le ha parecido bien". "Después he encontrado buena gente, pero ninguna relación ha progresado. " "He aceptado no tener pareja."

\section{Evolución}

La paciente inicia el tratamiento terapéutico en septiembre de 2004, permaneciendo de baja laboral desde junio. Reconoce que desde mayo lo estaba pasando mal. Tenía que interrumpir el trabajo en su consulta para llorar.

Había pedido el mes de junio de vacaciones para terminar el camino de Santiago, iniciado el año anterior. Cuando le dan la baja, sus amigas la animan a irse con ellas al Camino, y así lo hace. Al principio se siente culpable, no puede disfrutar, está llena de remordimientos por no estar trabajando. Su amiga la apoya y logra sentirse mejor. Al volver del viaje es capaz de hacer unos arreglos en su casa que había programado con anterioridad, y de nuevo se siente culpable por el mismo motivo. "Me ha costado mucho darme cuenta de que no estaba en condiciones de trabajar", comenta Carmen.

El aceptar que tiene que seguir un tratamiento farmacológico y psicoterapéutico, le supone reconocer que está enferma, que no puede superar sin ayuda su problema, y aceptar una situación de dependencia terapéutica que percibe como una amenaza a su autonomía personal. 
Estos sentimientos también la invadieron cuando estando trabajando como médico fuera de su ciudad estuvo de baja, ingresada por una infección gastro-intestinal. En aquella ocasión no quería que la atendieran y mucho menos quedarse hospitalizada. Pretendía seguir trabajando, aunque tuviera que interrumpir las consultas con frecuencia debido a su malestar. Le costaba aceptar los cuidados y la ayuda de sus familiares durante el ingreso.

"Todavía mi padre me dice”: “Qué haces aún de baja? Tienes que poder”. "Ahora sé que a veces no se puede." "El lema de mi padre siempre ha sido": "Hay que hacer las cosas bien y rápido, cueste lo que cueste"."

En el trabajo dedica mucho tiempo a cada paciente, prescinde de pedir ayuda, hace todas las gestiones personalmente, sale muy tarde, renuncia a actividades de formación profesional por falta de tiempo y se siente impotente para lograr hacer todo lo que se exige a sí misma: "Hacer todo bien y rápido... los dos pilares de mi vida son el trabajo y la familia".

En el ambiente familiar va por delante de los todos, cubriendo lo que ella entiende que son las necesidades del otro sin que se lo pidan. Los fines de semana se da por hecho que está disponible para ocuparse de los padres y de las necesidades que puedan tener otros familiares. Al final del día de fiesta se pregunta: “QQué he hecho yo?”. En ocasiones le pesa esta situación. Ha aprendido de su madre a estar disponible para los demás, sin saber decir que no. Le resulta difícil marcar una frontera entre su parcela y la del otro. "Siempre pienso que los otros necesitan más que yo", manifiesta. No pregunta a los demás que quieren o necesitan antes de hacer algo para ellos.

En noviembre de 2004, Carmen se encuentra mejor, pero le da miedo volver al trabajo y pasarlo mal. "Me va a resultar muy difícil dar a los pacientes lo que me piden sin fundamento, me queda someterme a esa manipulación externa o enfrentarme al enfermo". "Me estoy replanteando la profesión de mi vida, tal vez tenga una excesiva dedicación con unos resultados insuficientes para el esfuerzo".

Al volver al trabajo, Carmen se esfuerza en organizar su jornada laboral, y delega funciones que antes hacía ella misma, en el personal correspondiente. Al principio se siente culpable, egoísta, pero persiste en el intento. Logra algunos cambios de actitud, de horario, llegando a ser capaz de dejar algunas gestiones para el día siguiente, así como salir a su hora del trabajo.

En la parcela familiar logra dejar de ir a casa de los padres todos los fines de semana sistemáticamente, y consigue poder quedarse en su propia casa ocupándose de sus cosas o descansando. Entiende que se excede en la atención a su sobrino, dificultando el aprendizaje de su hermana pequeña de su papel de madre Carmen se esfuerza en organizar su jornada laboral y delega funciones, que antes ella hacía, en las personas a las que les corresponde. Al principio se siente culpable, pero persiste logrando algunos cambios. Puede dejar gestiones para mañana y no se siente tan culpable por ello como para no poder salir con las amigas, ir al cine o divertirse. Expresa que se siente útil y valiosa cuando los demás le piden algo, pero a ella le cuesta pedir porque se siente en deuda con el otro.

Finalmente, acepta que se encuentra demasiado mal para poder trabajar y pide la baja durante 3 días debido a un fuerte enfriamiento. Cada vez que logra introducir un cambio en su vida y tiene mejor estado de ánimo propone un distanciamiento de las sesiones de psicoterapia.

En junio de 2005 solicita un traslado a otro centro y pasa unos días de vacaciones. Al volver, comenta que no se atreve a decirle a su padre que sigue asistiendo a terapia. Según él ella no lo necesita porque es muy fuerte.

Después de disfrutar de los días de vacaciones que le quedaban, decide dejar la terapia por considerar que se encontraba bien. Finalmente es dada de alta, logra un cambio en su puesto de trabajo y se encuentra muy motivada para volver a intentarlo "por segunda intención". 


\section{Estudio psicométrico}

Se confirma la existencia de síntomas moderados de ansiedad y depresión, así como de tipo obsesivo y de somatización. En su personalidad destaca el predominio de los aspectos sanos, con rasgos moderados de tipo fóbico y obsesivo.

\section{Tratamiento}

Se le indicó tratamiento farmacológico (Venlafaxina y Trazodona) y psicoterápico individual, y se recomienda que permanezca de baja laboral hasta alcanzar una mejoría suficiente.

Llama la atención las dificultades de Carmen para poder estar de baja laboral sin sentirse culpable, darse permiso y reconocer su derecho legítimo a tener problemas personales, hablar de sus sentimientos negativos, aprender a relajarse...y para poder adquirir estrategias adaptativas de autocuidado, según reza el epitafio clásico: «médico, cúrate a ti mismo».

\section{CONCLUSIÓN}

De las seis principales causas que producen pérdida de calidad de vida y años con discapacidad en el grupo de personas de 15 a 44 años de edad, cuatro son trastornos mental: depresión, abuso de alcohol, esquizofrenia y trastorno bipolar, por lo que su diagnostico precoz y el tratamiento eficaz deben constituir una prioridad asistencial al tratarse de un grave problema de Salud Pública.

Frente al modelo de formación tradicional en Salud Mental, como se realiza habitualmente en las facultades de Medicina, que han demostrado ser poco eficientes para formar a los médicos generales para la detección y orientación terapéutica de los pacientes con trastornos mentales, la Asociación Mundial de Psiquiatría ha propuesto recientemente un modelo formativo basado en el aprendizaje de competencias y habilidades específicas en Salud Mental. Este modelo tiene 3 componentes:

1. Evaluación de las necesidades formativas en Salud Mental por profesores cualificados y capacitados.

2. Plan de estudios que incluyen una serie de módulos estructurados para ayudar a los profesionales sanitarios a abordar los distintos trastornos mentales.

3. Aplicación de los mismos.

Desde nuestra perspectiva, seria conveniente añadir un cuarto paso de evaluación de resultados y una posterior aplicación con las mejoras implementadas.

De igual manera, cobra especial importancia la formación en Salud Mental en los médicos del trabajo, y la inclusión de variables psicológicas en la vigilancia de la salud de los trabajadores.

El trabajo en su más amplio sentido realiza una función organizadora en el psiquismo; como referente individual y como grupo de pertenencia. La profesión aporta signos de identidad, delimitando, ayudando a la presentación del "sí mismo" (en nuestro medio utilizamos el "soy..."). Es por lo tanto un componente en la representación psíquica del "sí mismo", del self de primera magnitud.

El empleo, el puesto de trabajo aporta una ordenación temporo-espacial al Yo consciente. Horarios, organización de la semana (días hábiles y festivos) y del año (períodos laborales y vacacionales). Es por lo tanto una herramienta-eje en la gestión de nuestro tiempo.

Los sentimientos de incapacidad, desvaloración laboral son constantes en los procesos depresivos amenazando y frecuentemente dañando la identidad profesional. 
Desde un punto de vista preventivo, cuando existe una etiología relacionada con el trabajo, la prevención primaria deberá focalizarse en realizar los cambios organizacionales necesarios para "prevenir en el origen", tal y como señala la Ley de Prevención de Riesgos Laborales. Las intervenciones sobre el paciente depresivo se plantean desde una prevención secundaria, dando herramientas para el manejo y control de las situaciones estresantes y cambios en el estilo de vida. La intervención (o prevención terciaria) supondrá la ayuda directa a la persona para la recuperación de su salud, con los tratamientos farmacológicos y psicoterápicos señalados en apartados anteriores.

La habitual coexistencia de alteraciones psicológicas y somáticas en la persona con depresión hace necesario un enfoque psicosomático integrador, interdisciplinar ${ }^{21}$, para que no ocurra como se lamentaba Vesalio en el Renacimiento, que ya se quejaba del "lastimoso desmembramiento del arte de sanar...", por el propio desarrollo de las ciencias básicas y la consiguiente super-especialización asistencial. Para conseguir una asistencia de calidad es preciso la detección precoz y el tratamiento eficaz de los pacientes en el circuito asistencial y en su entorno laboral.

A modo de conclusión será importante recordar los siguientes aspectos:

Tabla II. El paciente depresivo en el trabajo

\section{Conclusiones:}

- La depresión es muy prevalente entre la población trabajadora y habitualmente no se reconoce ni se trata de forma eficaz.

- Cuando existe una etiología relacionada con el trabajo, la prevención primaria y secundaria serán especialmente importantes para su control.

- Si ya es necesaria una intervención, el tratamiento debe incluir medicación antidepresiva y psicoterapia. Si esta se realiza, la mejoría clínica es significativa entorno al $70 \%-80 \%$.

- La eficacia de los antidepresivos a largo plazo es de un $90 \%$ logrando una calidad de vida satisfactoria.

- La sintomatología depresiva puede ser foco de conflictos laborales e interpersonales, y a su vez, determinados factores psicosociales del trabajo son facilitadores de algunos aspectos depresiógenos.

- La depresión requiere una detección precoz y una orientación terapéutica para prevenir su posible cronificación y posibles complicaciones graves, como pueden ser las conductas de abuso del alcohol y/o de sustancias adictivas.

- Las depresiones mal llamadas "menores" o distimia, predisponen a la depresión mayor y se alternan con frecuencia a lo largo del tiempo.

- Un adecuado abordaje psicoterapéutico de forma temprana permite, en la mayoría de los casos, la reincorporación laboral, cumpliendo el trabajo un efecto protector y estabilizador en la persona.

- El abordaje biopsicosocial del problema debería incluir un apoyo organizacional adecuado, facilitando los recursos, programas asistenciales y la formación necesaria para la adquisición de competencias en el manejo y control de los estresores laborales, la prevención de los mismos y el seguimiento e intervención más efectiva sobre los casos detectados.

\section{BIBLIOGRAFÍA}

1. Mingote J.C.; del Pino P.; Huidobro A.; Gutiérrez D.; de Miguel I.; Gálvez M. El paciente que padece un trastorno psicótico en el trabajo: diagnóstico y tratamiento. Med Segur Trab 2007; 52 (208): 29-52.

2. Ramos J. y Cordero A. La melancolía. Gestación de la hipótesis secuencial. Grupo Aula Médica. Formación Continuada. Madrid 2005.

3. Murray C.S.L.; López A.D. The global burden of disease: A comprensive assesment of mortality and disability from diseases, injuries and risk factors in 1990 and projected to 2020. Cambridge, MA. Harvard University Press 1996.

4. US Surgeon General. Mental Health: A report of the surgeon general 1999. Disponible on line en: www. surgeongeneral.gov/library/mentalhealth/chapter2/sec2_1.html\#epidemiology

5. INSHT. Ministerio de Trabajo y Asuntos Sociales. VI Encuesta Nacional de Condiciones de Trabajo 2007. Disponible on line en: www.mtas.es/insht.

6. Coyne J.C. Toward and interactional description of depression. Psychiatry 1976; 391: 28-40. 
7. Merikangas K.R. Assortive mating for psychiatric disorders and psychological traits. Arch Gen Psychiatry 1982; 39: 1173-1180

8. Karasek R.A. Job demands, job decision latitude, and mental strain: implications for job desing. Administrative Science Quarterly 1979; 24: 298-308.

9. Broadbent D.E. The clinical impact of job design. Br J Clin Psychol 1985; 24: 33-44.

10. Kawakami N., Araki S., Kawashima M. Effects of job stress on occurrence of major depression in Japaneses industry: a case-control study nested in a cohort study. J Occup Med 1990; 32: 722-725.

11. Bromet E., Dew M., Parkinson D et al. Effects of occupational stress on the physical and psychological health of women in a microelectronics plant. Soc Sci Med 1992; 34: 1377-1383.

12. Chevalier A., Bonenfant S., Picot M-D. et al. Occupational factors of anxiety and depressive disorders in the French National Electricity and Gas Company. J Occup Environ Med 1996; 38: 1098-1107.

13. Paterniti S.; Niedhammer I.; Lang T.; Consoli S.M. Psychosocial factors at work, personality traits and depressive symptoms: longitudinal results from the GAZEL Study. The British Journal of Psychiatry 2002; 181: 111-117.

14. Niedhammer I., Goldberg M., Leclerc A., et al. Psycholsocial factors at work and subsequent depressive symptoms in the Gazel cohort. Scand J Work, Environ Health 1998; 24: 197-205.

15. Kawakami N., Haratani T., Araki S. Effects of perceived job stress on depressive symptoms in blue-collar workers and electrical factory in Japan. Scand J Work, Environ Health 1992; 18: 195-200.

16. Agerbo E., Gunnell D., Bonde J.P., Mortensen P.B., Nordentoft M. Suicide and occupation: the impact of socio-economic, demographic and pshychiatric differences. Psychol Med 2007; 37(8): 1131-1140.

17. Arrizabalaga P., Valls-Llobet C. Mujeres médicas: de la incorporación a la discriminación. Med Clin (Barc). 2005; 125(3): 103-7.

18. Liu P.M., Van-Liew D.A. Drepression and Burnout. En Mental Health and Productivity in the Workplace: A handbook for organizations and clinicians. San Francisco, CA, US. Jossey-Bass 2003; Cap.22: 433-457.

19. Maslach C, Jackson S. Maslach Burnout Inventory Manual. Palo Alto: Consulting Psychologists Press, 1986.

20. Moreno B, Gálvez M, Garrosa E, Mingote C. Nuevos planteamientos en la evaluación del burnout. La evaluación específica del desgaste profesional médico. Aten. Primaria 2006; 38 (10): 544-549.

21. Mingote J.C., Mingote B. La depresión: una enfermedad como otra cualquiera. Díaz de Santos. Madrid 2008 (En prensa). 


\section{ANEXO: DIAGNOSTICO DIFERENCIAL}

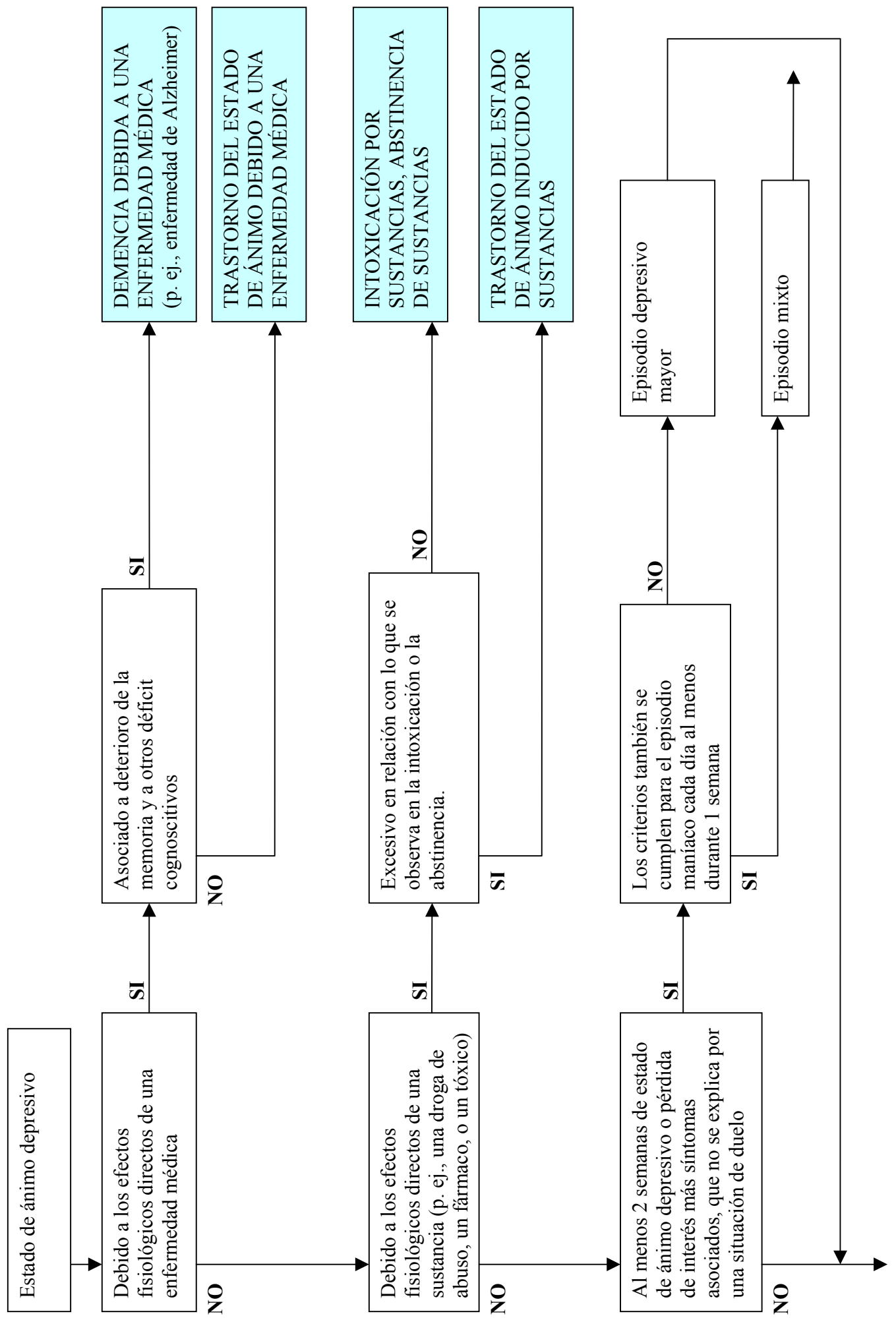




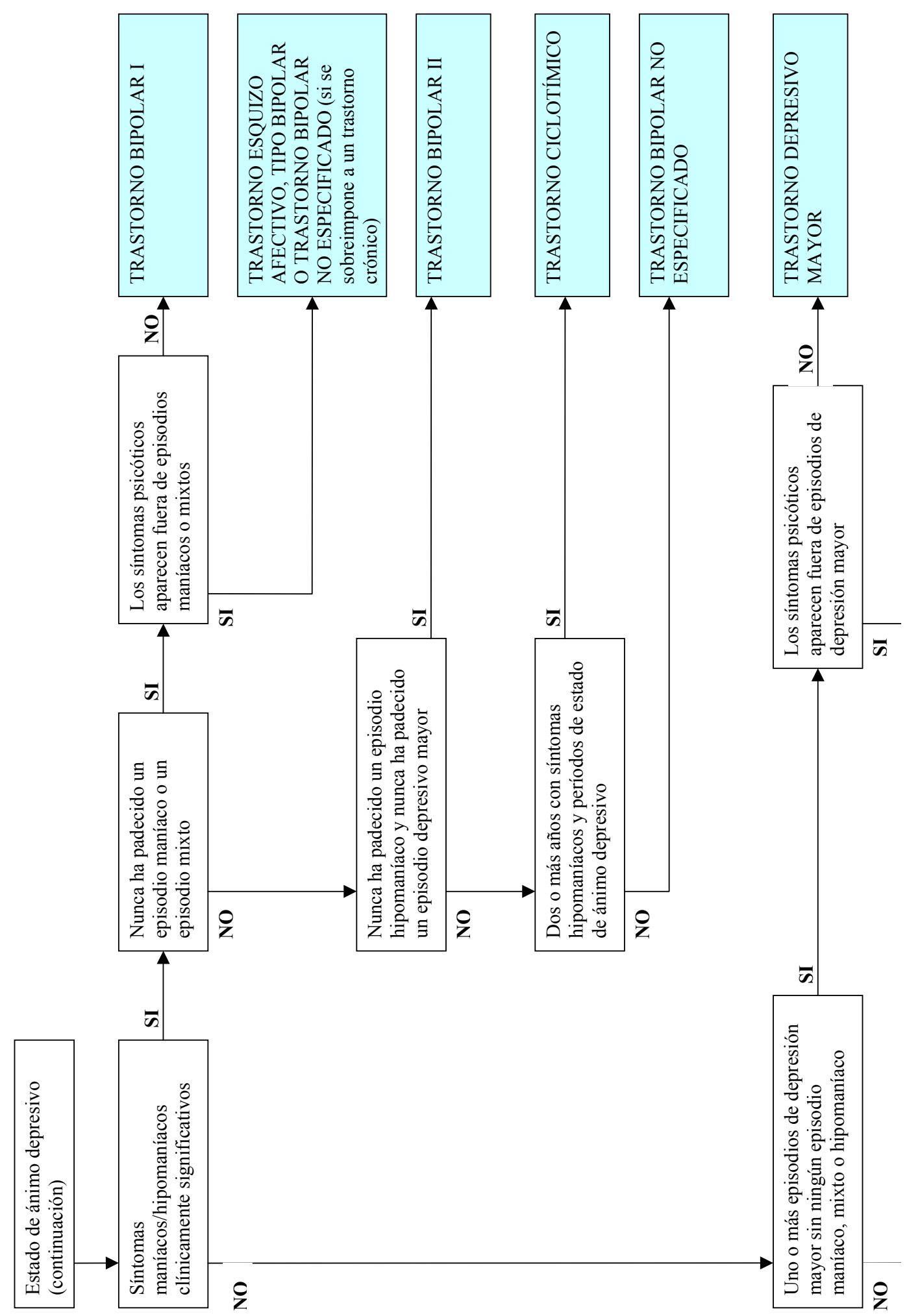




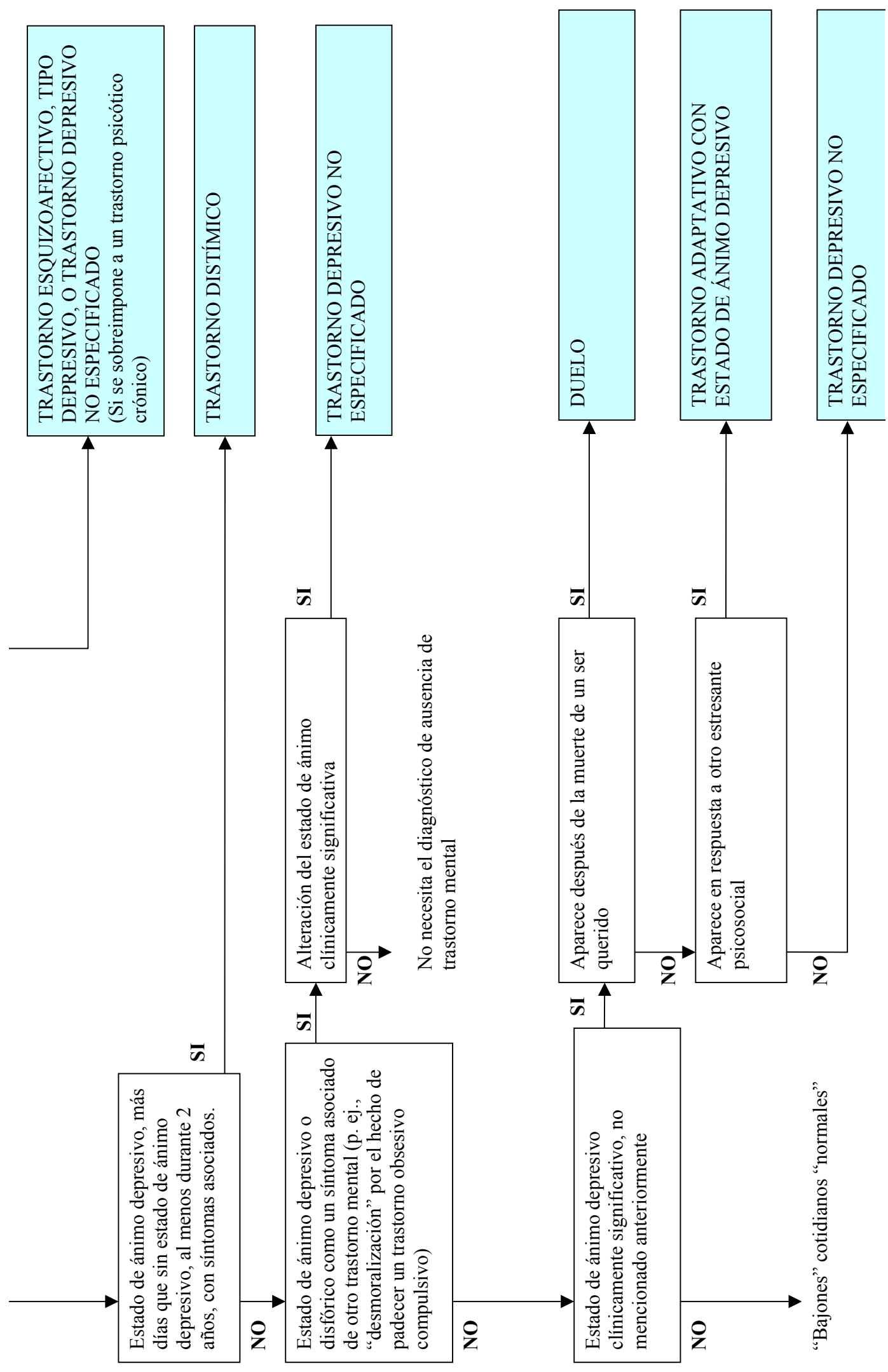

